\title{
SCISSOR-HINGED DEPLOYABLE STRUCTURES SUPPORTED PERIMETRALLY ON RECTANGULAR BASES
}

\author{
M. J. FREIRE-TELLADO (1); M. MUÑOZ-VIDAL (2); J. PÉREZ- \\ VALCÁRCEL (3).
}

\author{
Title, affiliation, address, and e-mail of all Author(s) \\ (1) Ph.D. Architect, Professor, CEACA Department, University of A Coruna. Address: ETS Arquitectura, Castro Elviña \\ s/n, 15071 A Coruña, Spain. manuel.freire.tellado@udc.es \\ (2) Ph.D. Architect, Professor, CEACA Department, University of A Coruna. Address: ETS Arquitectura, Castro Elviña \\ s/n, 15071 A Coruña, Spain. manuel.munoz@udc.es \\ (3) Ph.D. Architect, Full Professor, CEACA Department, University of A Coruna. Address: ETS Arquitectura, Castro \\ Elviña s/n, 15071 A Coruña, Spain. juan.pvalcarcel@udc.es
}

\begin{abstract}
Editor's Note: This space reserved for the Editor to give such information as date of receipt of manuscript, date of receipt of
\end{abstract} revisions (if any), and date of acceptance of paper. In addition, a statement about possible written discussion is appended.

DOI: Digital Object Identifier to be provided by Editor when assigned upon publication. https://doi.org/10.20898/ j.iass.2020.204.009

ABSTRACT

Deployable bar structures on a rectangular base present the problems of closing the ends and longitudinal stabilization. Some proposals have been made to resolve them, mainly by creating mouths, but their application to composite geometries is problematic.

On-the-bias deployable structures adequately solve both problems as they are supported around their entire perimeter, have a strong three-dimensional operation and only use load-bearing bars. The text analyses this type of deployable structures, their requirements and possibilities.

A study on the combination of polar and translational units in a single deployable structure is then carried out, focusing on the study of on-the-bias deployable structures with perimeter polar modules that allow the effective height of the designs to be increased. Finally, the architectural possibilities of the system are studied, which make it possible to resolve overhangs, vertical openings in the roof, and $T$ - or L-shaped ground plans, guaranteeing the modularity of the system.

Keywords: Lightweight structures, deployable structures, scissor-hinged mechanism, deployment sequence, conceptual design, morphology.

\section{INTRODUCTION}

Scissor-like deployable structures are strong assemblies formed by pairs of bars linked at an intermediate point by scissor-type joints - called blades - and joined to similar blades at the ends of the bars by means of hinges. The resulting system can be arranged in one, two, three or four directions and has the property of being able to be deployed quickly: once built, in a folded position, it forms a compact package of bars that, taking advantage of the system's degrees of freedom, can be deployed and cover a wide area. Once the desired configuration has been obtained, the possibility of movement is blocked and the unit functions as a structure.
At present, although only a few examples have been built, several proposals have been made and the bibliography on them is becoming abundant. The designs that have been created usually consist of lightweight roofs for buildings and also shelters, common service buildings, or temporary housing in emergency situations, although there are also some that do not have a defined use. In the case of our team, its study forms a part of the search for solutions for buildings to be used in emergency situations: it is important to note that, according to UNHCR data, refugee records are broken year after year. Within the range of emergency structures, this system is particularly suitable for institutional buildings. 


\section{THE CLOSING OF THE EXTREMITIES}

The described approach justifies the interest in rectangular layouts, due to the organizational flexibility that they allow and the necessity to solve the entire building envelope.

In the field of deployable structures, although there are many theoretical proposals, built examples are scarce. This may be why there are not many cases that include the solution of all the building enclosures. In dome solutions this aspect is not very relevant due to the reduction in the free height at the edges: this is the case of the cover of the swimming pool of San Pablo de Sevilla de Escrig, Valcárcel and Sánchez [1], composed of two rectangular sailor vaults. However, on cylindrical roofs or on gable roofs of a certain size, the solution to the enclosures of the front ends inevitably conditions the viability of the building, given that without them the building is not closed, significantly restricting its possibilities of use.

Completely closing the building requires that the deployable structure is supported along the entire perimeter or that an auxiliary system is used to complement the deployable system in the areas left open by the structure.

Within the first option, Sánchez Cuenca [2] solves the problem using a design in the shape of a pyramid based on translational modules, and approaches the solution in three other proposals in which the corners remain open.

De Temmermann [3] also addresses the problem: in his doctoral thesis - p. 177 - and under the title 'Case study 2: A Deployable Barrel Vault with Polar and Translational Units on a Two-way Grid' where he proposes a small deployable barrel vault combined with Lamella-type structures to resolve the extremities.

Another possibility is to use modules that reduce the height of the extremities. This is the option chosen by Hernández Merchán and Zalewski in the TaraTara Itinerant Exhibitions Pavilion [4], where, based on the ESTRAN 1 prototype [5] they create an opening that reduces the size of the extremity, and stabilizes the whole structure.

This same idea was explored by F. Escrig [6] combining cylindrical vaults with spherical edge caps (p.67) built with polar modules (cover for the swimming pool of the nautical club of Almeria, p.68), and also with conical extremities (p.69).
Assuming the change in dimensions introduced at the ends of the layout, this possibility has also been investigated, combining cylindrical surfaces resolved with polar modules with conical shells of translational modules. Its application to cylindrical Tudor profile deployments was studied, meshes that our team had previously resolved [7], combining them with cone trunks. The system was successfully solved, first at the graphic level, by solving the layout and folding of the system. The kinematic conditions were then experimentally verified using a series of bar models (Figure 1) constructed with $4 \mathrm{~mm}$ thick methacrylate bars and polylactic acid nodes (PLA).

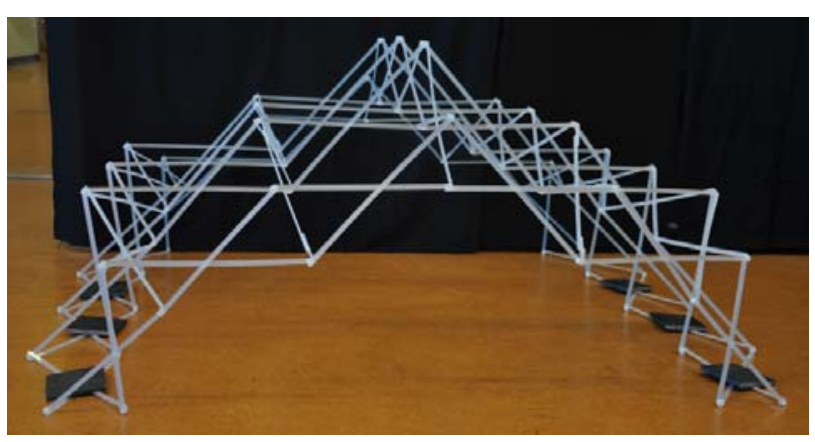

Figure 1: Tudor deployable grid with arch

The assembly consists of a series of vertical arcs parallel to each other whose dimension is reduced at the ends - bearing modules - combined with trasverse scissor systems that stabilize the arcs bracing modules-. The layout of the mouthpiece is based on the form described in [8], but in order to comply with the conditions for folding the whole assembly, it was decided to keep the planes of the transverse blades constant (in blue in Figure 2; compare with [5] Fig. 5), which necessitated the use of several homothetic centres. Therefore, the arch of the mouth is not completely homothetic to the base arch: the assembly has three homothetic zones linked by two intermediate zones $(\mathrm{k})$, whose lengths are maintained. The non-homothetic bars become proportionally longer, and are converted into the supports for the assembly when the bars are folded, which in this situation is only supported at four points. However, this limitation is of little significance, due to the minimum stresses produced when the structure is folded.

In addition, the bracing blades $(\mathrm{k})$ require different lengths in the folding and unfolding positions, which necessitated the use of symmetrical telescopic rods made with sliding sections at both ends, in order to allow for the necessary length 
adjustments without modifying the connecting point between the rods. The differences in length increase when the mouth is closed, which conditions the applicability of the solution.

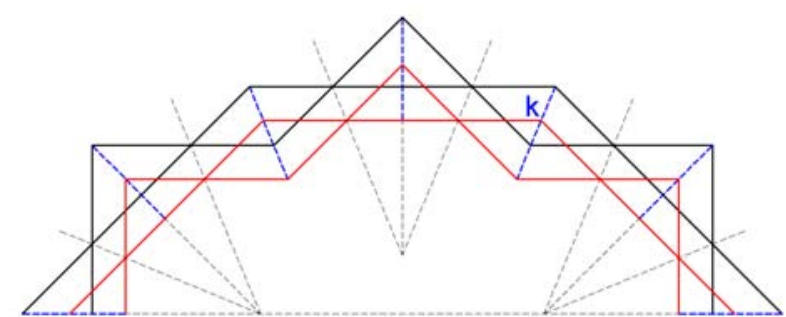

Figure 2: Elevation of Figure 1

\section{3. 'ON-THE-BIAS' DEPLOYABLE GRIDS}

\subsection{Definition and development}

In the previous lines the limitations of the two-way deployable meshes have been explained with the scissors arranged in the plane of the section (it is also possible to place the meshes in the vertical planes of the enclosure and in the roof skirts as described in [9], although the solution is only of interest for small dimensions). In comparison to these, the on-the-bias arrangement (rotated $45^{\circ}$ with respect to the sides) of the meshes allows support throughout the perimeter of the building without altering the floor plan. The fundamental characteristic of this solution [7] is that the scissors of both directions are load-bearing, eliminating the dichotomy between load-bearing and bracing units. The foldability of the solution requires that the movements of the nodes during folding and unfolding are contained at the intersections of the bar planes in both directions, which is normally the vertical direction. This condition requires the use of translational modules - also called oblique modules - which will have a constant edge if both directions are orthogonal and the inclination of the guidelines is constant. In this case, if the bars are always placed in the same way, an enclosure with two gables is obtained with support on two parallel sides, while by inverting the arrangement of some bars, a four-gable enclosure is obtained, supported along its entire perimeter (Figure 3).

If the slope varies, as occurs in situations with curved shapes, compliance with the conditions of foldability ${ }^{1}$ make it necessary to modify the edge:

\footnotetext{
${ }^{1}$ In order for the mesh to be foldable, the sums of the distances between the articulated nodes connecting
}

as the horizontal projection of the separation between modules remains constant, as the slope of the line joining the centres of ellipses -the guideline- changes, the intersection of this line is modified with the ellipse that expresses the condition of compatibility of the folding process, which we will refer to as the folding ellipse. As this intersection defines the position of the central articulation of the scissor, compliance with the previous condition means it is necessary to modify the edge.

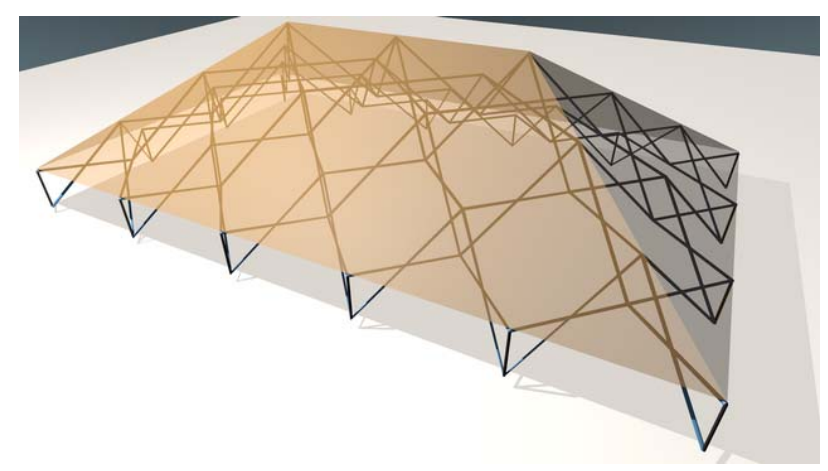

Figure 3: Hipped Roof in Bias Deployable Grid

With the appropriate bars -those that meet the conditions of foldability- a deployable structure is created whose envelope is a cylindrical surface with support on two parallel sides and open fronts or with the same bars but with different placement- a deployable structure in the shape of a rectangular pavilion vault with support around the perimeter, so that the roofing material also becomes an enclosure (Figure 4).

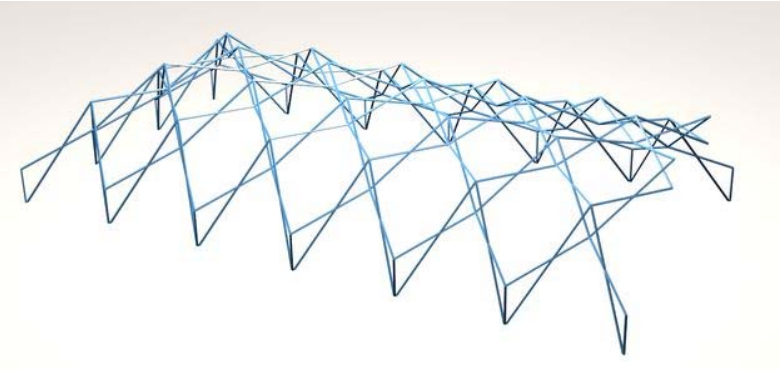

Figure 4: Bias Deployable Grid Shaped as Semi

the blades and the central scissor-type joint must be equal for each scissor. This condition is equivalent to requiring that, in $2 \mathrm{D}$, the scissor joints be located on an ellipse whose focal points are the connection nodes between the blades. In 3D, the scissor type joints have to be on the ellipsoids of revolution of vertical axis derived from the rotation of the anterior ellipse. 


\section{Rectangular Pavilion Vault}

If, with the same system, we start with a square ground plan, we obtain deployable structures with a pyramid envelope or a cloister corner vault, which can be a good option to temporarily cover cloisters and patios in historical buildings (Figure 5).

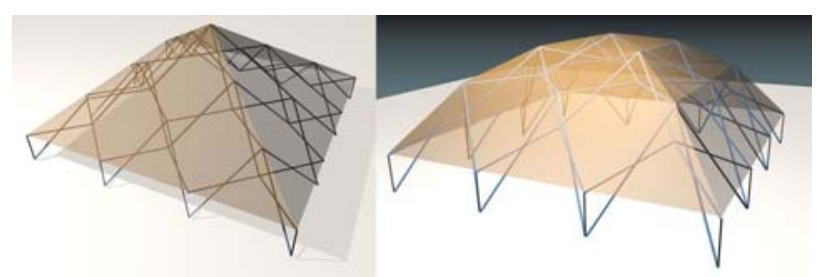

Figure 5: Bias Deployable Grids with a Square Ground Plan

Both previous geometries can be combined, using a generator formed by curved and straight sections, which will also be deployable if it is adjusted to the folding conditions (Figure 6).

An initial deployable layout can also be transformed by modifying the slopes of the initial and ridge blades to suit the specific requirements of the project; if this is done in compliance with the folding conditions, the result will still be deployable (Figure 7).

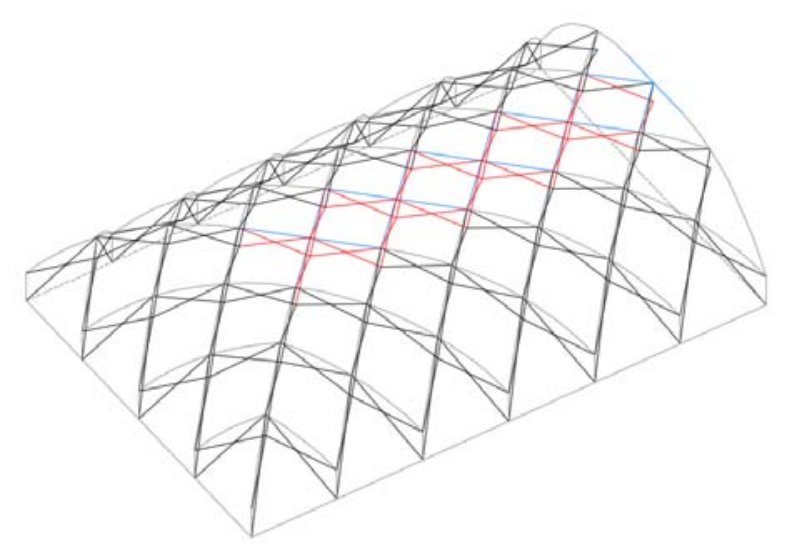

Figure 6: Bias Deployable Grid Shaped as Pavilion Vault with a Central Flat Section

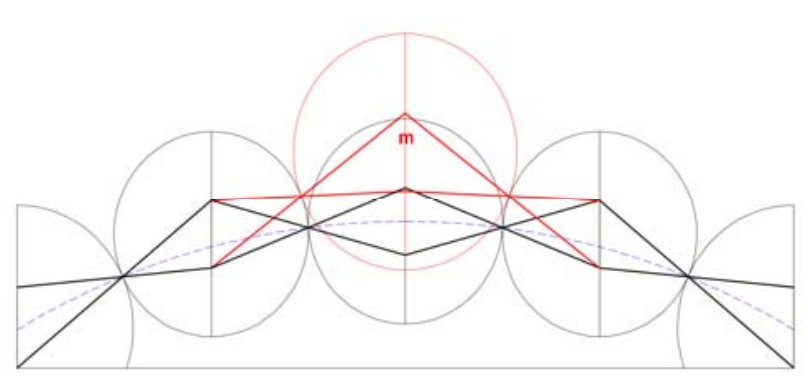

Figure 7: Modification of the Ridge Scissors Based on Foldable Ellipses.

These modifications do not have to affect all the nodes in the same situation nor affect all of them equally, which allows for interesting modifications.

\subsection{Model in the shape of a four-gabled roof}

In order to experimentally corroborate the deployability of these constructions, two and fourway kinematic roof models were constructed, demonstrating the versatility of the solution. Both were assembled from systems of deployable frames placed on a rectangular ground plan with an inclination of $\pm 45^{\circ}$ with respect to the longitudinal axis and a slope in true magnitude of $25^{\circ}$. For this purpose, 48 translucent methacrylate bars were laser cut and 36 PLA nodes were produced using a $3 \mathrm{D}$ printer (Figure 8), of the type known as eccentric bar axis nodes -[10] p. 144-. The assembly process is very simple, as the models are constructed with only two types of bars that are also symmetrical, only differing in the assembly of the extremities. Four more vertical bars were added at the corners to each ensemble to fix the position of the model once deployed [7]: in deployable structures it is important to ensure that the supports are not made on a single bar, in order to limit the bending that can occur.

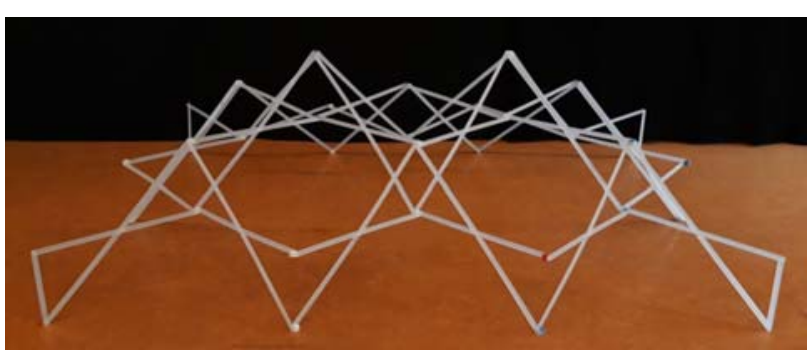

Figure 8: Model of Bias Deployable Grid with Hipped Roof.

The folding and unfolding processes carried out suggest the use of cranes as auxiliary equipment for 
unfolding or, after horizontally displacing the bars on one side, putting of a series of perimeter tensers in place - in practice, winches are used - which can also be used to pre-tense the structure to a certain degree once the pre-set unfolding position has been reached.

Two tests have been carried out with the covering material (Figure 9), which have highlighted the need to brace the corner scissors, as the forces introduced by the fabric displace them from their theoretical position.

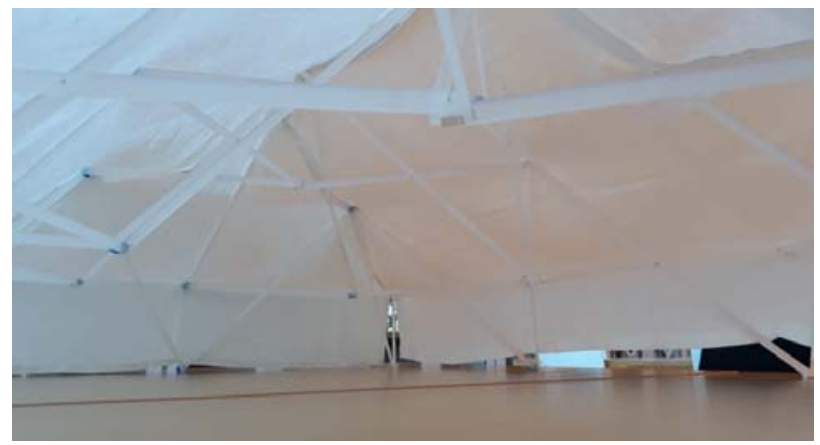

Figure 9: Inside View of the Model.

Two sets of dynamic calculations of the proposed structure with the ARTIC programme [11] have also been carried out in order to verify its feasibility and structure. The calculations show the good structural behaviour of the whole, especially when horizontal displacement of the nodes is prevented or perimeter fastening bars such as those observed in the corners of the model are installed.

\subsection{Model with rectangular pavilion vault shape}

The second type of model that was made corresponds to a deployable structure with a curved shape, with a rectangular pavilion vault-shaped enclosure with a lowered profile (Figure 10). In this case 120 bars and 78 nodes with slightly smaller dimensions were used. The bars are grouped into only six types, but the assembly is now more demanding due to the asymmetry of the elements. This requires careful organization at the assembly level, which of course will also be necessary in the construction process.

The assembly and testing of the model revealed the internal redundancy of the solution: although scissor mesh systems have a degree of freedom which makes deployment possible - they have more internal constraints than precise meshes, derived from transverse linkage. It was experimentally proven that the folding and unfolding process is properly executed even when internal constraints are eliminated, a fact that provides peace of mind in the event of the failure of any element during assembly or disassembly.

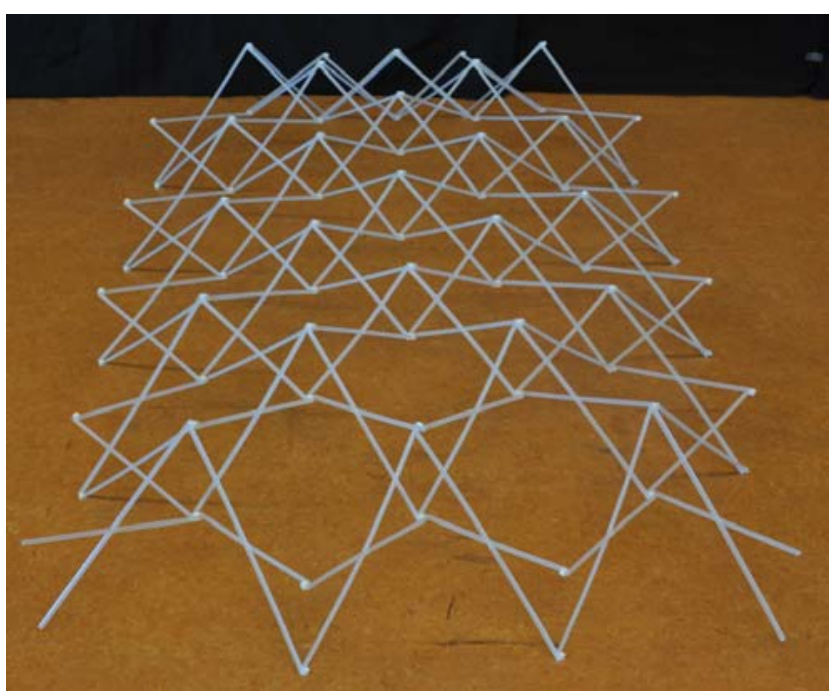

Figure 10: Model with Lowered Rectangular Pavilion Vault Shape.

In both models, and as a consequence of the translational module used, only the perimeter nodes touch the ground, which despite being advantageous on the one hand because it facilitates the deployment, on the other hand obliges the bars and nodes to work from the beginning.

It also became evident that the system is perfectly compatible throughout deployment, without stresses due to incompatibilities, remaining in balance at any point in the deployment process. This factor meant that no stabilizing bar was used as can be seen in the photos. In reality, however, it would be necessary to introduce auxiliary perimeter rods linking the lower and upper points of the verticals in order to ensure correct deployment, reduce mechanical stress, and increase the rigidity of the assembly.

The deployed situation revealed the difficult position of the corner blades in this model, which do not touch the ground. This may be a consequence of the development of the corner torsion mechanism, although the scissor remains loose, only stabilized by the weak transverse stiffness provided by the junction nodes (Figure 
11).

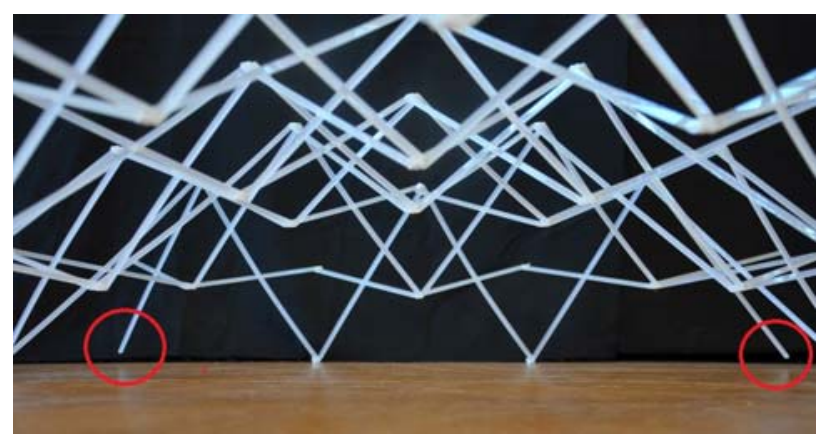

Figure 11: Nodes Raised from the Floor

In this situation there are two possible alternatives: bevel the ground plan and remove the corner scissors, or, on the contrary, fix them in position by adding new bars. The choice of the solution depends on the specific architectural proposal to be made, but in general it does not always have to be admissible to eliminate the corner blades.

These scissors can be fixed with bars with a intermediate lockable link, but in this way only one node would be fixed. If the aim is to fix the two corner nodes in place, the solution is to use a system of scissors arranged at $45^{\circ}$, coinciding with the edges of the ground plan.

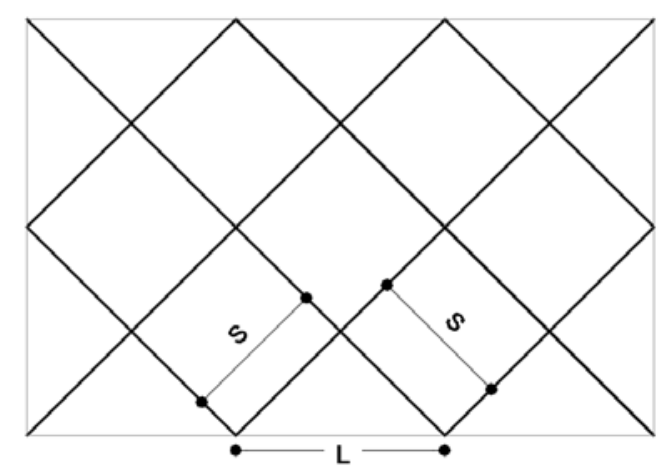

Figure 12: On-the-Bias Deployable Structure: Ground Plan

Due to the layout conditions on the ground (Figure 12), the horizontal distance that must be spanned by the edge blades is $L=s / \cos 45^{\circ}$. In addition, the folding conditions must be met, which means that the scissor-type joint must be on the surface of the vertical main axis revolution ellipsoid whose focal points are the bar connection end nodes. Both conditions cannot be met with a single blade, meaning it is necessary to use composite scissors in order to meet both conditions simultaneously (Figure 13).

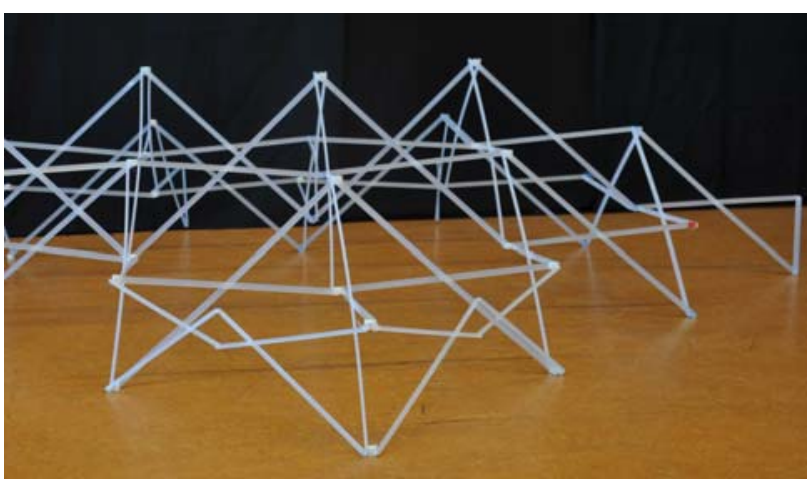

Figure 13: Stabilization of the Corner Nodes Using $45^{\circ}$ Compound Units

The term 'composite scissor' has been used, instead of the term 'double scissor' in order to overcome the ambiguity arising from the terms used by other authors, but which have other meanings. For example, Begiristain [10] p.82, uses the terms 'double scissor' or 'multiple scissor' to refer to the deployable structures of Pérez Piñero with two vertically superimposed scissors, while De Temmerman, [3] p.138- uses it to refer to compound blades.

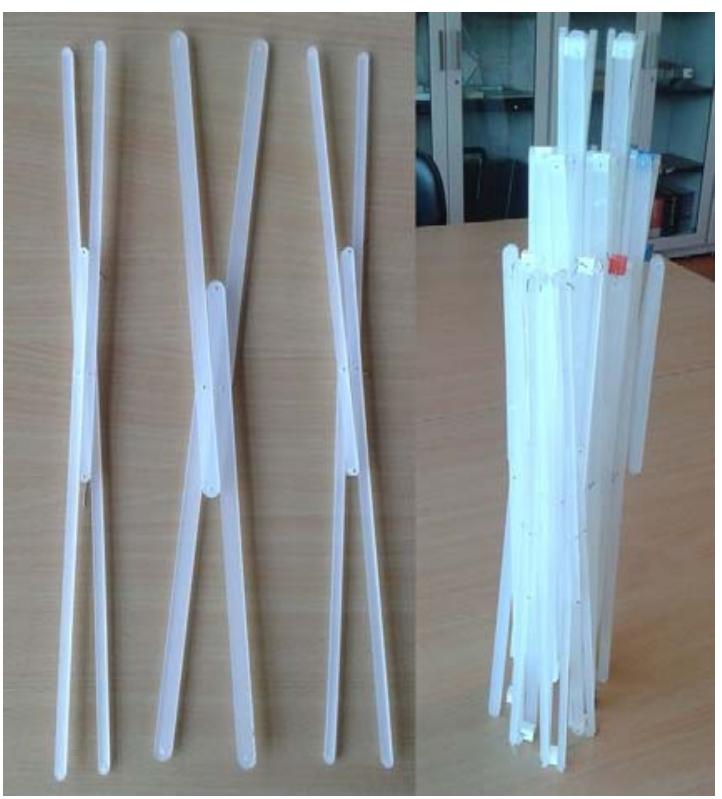

Figure 14: Folding of Compound Scissors, and Complete Folded Package Including these scissors.

To facilitate execution, these bars have been built with their components located on two adjacent 
planes. As can be seen in the image (Figure 14), the transversal width of the bars conditions the closing of the solution. However, this limitation does not affect the compactness of the folded bar package.

Although in order to fix the corner nodes it is only necessary to place composite scissor units on the end spans, the solution can be extended to the entire perimeter in order to facilitate completing the covering of the deployable structure, and to create a perimeter bracing band.

In addition, as the double scissors in this direction are symmetrical, it is possible to adjust the position of the intermediate node - within the limits imposed by the folding conditions - in such a way that it is at the same height as the point where the blade start. (Figure 15).

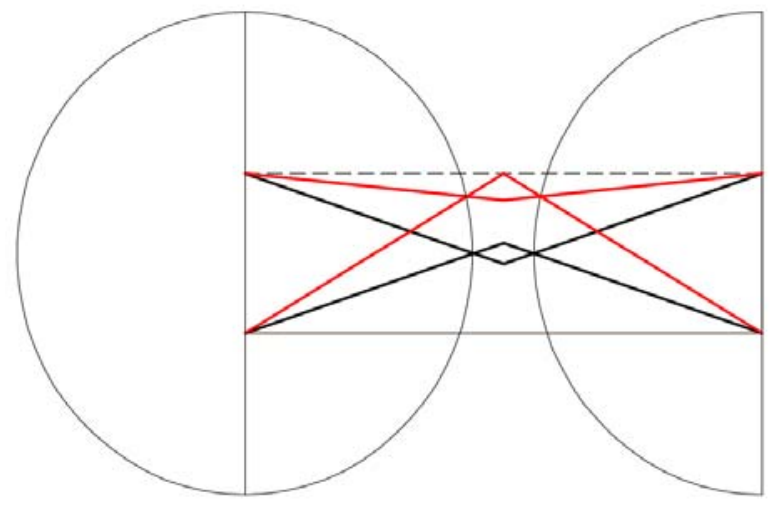

Figure 15: Layout of Lateral Compound Blades Based on Foldability Ellipses.

Using the previous system, the overall design can be improved by obtaining a new perimeter support for the covering material or by facilitating the organization of the entrances (door opening) (Figure 16). The same system can be used to solve the edges of openings in the roof formed by removing blades from the deployable structure.

In the same way that it is possible to modify the slope of the deployable at the ridge, it is possible to modify the slope at the edges to ensure that the starting section of the deployable structure can be closed with a single blade, for which the minor axis of the foldability ellipse corresponding to the starting point measures L.

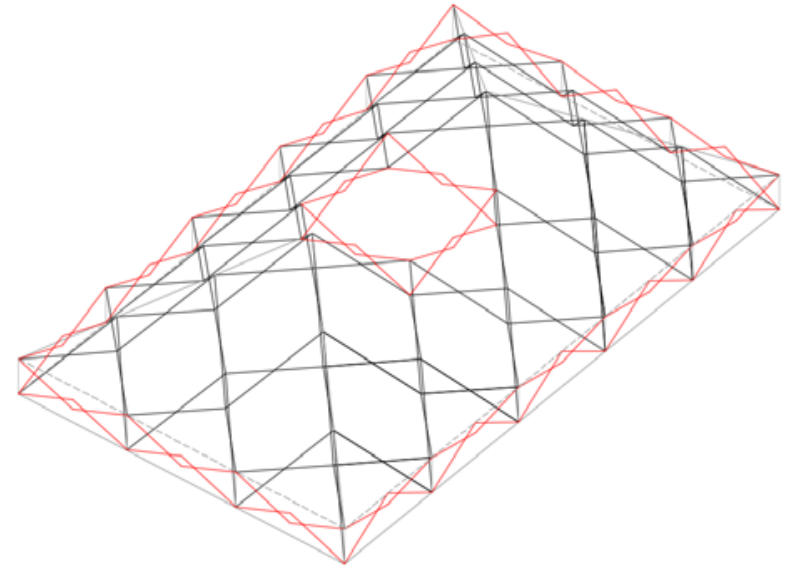

Figure 16: On-the-Bias Deployable Structure with Perimeter Bracing (two variations are shown) and an Upper Opening which is Also Braced.

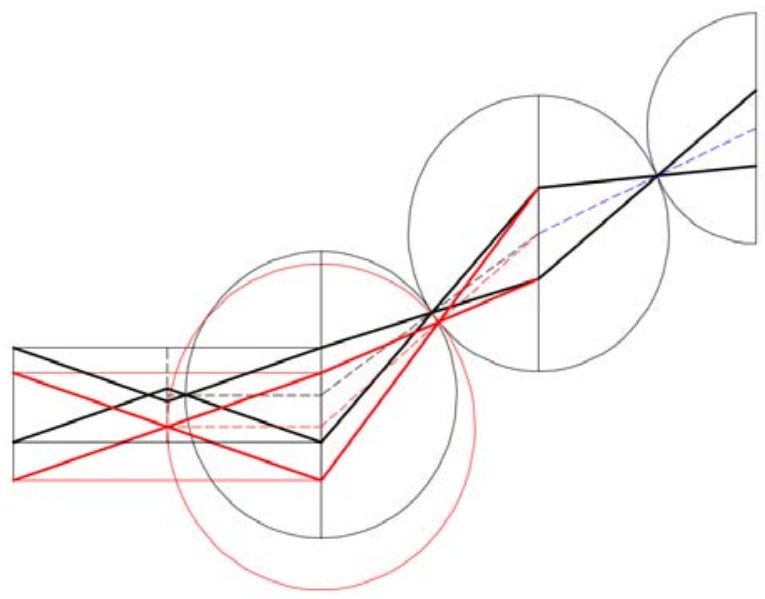

Figure 17: Correction of the Initial Slope.

The solution can be obtained by iterating graphically on the lowered paths (Figure 17) or by solving the non-linear equation system that expresses the two conditions of distance from the intermediate nodes to the edge nodes, and the obligatory straight line of the bars of the blade. It also introduces a break in the overall design, regardless of whether it is applied to a deployable structure with four gables, or to a rectangular pavilion vault structure. 


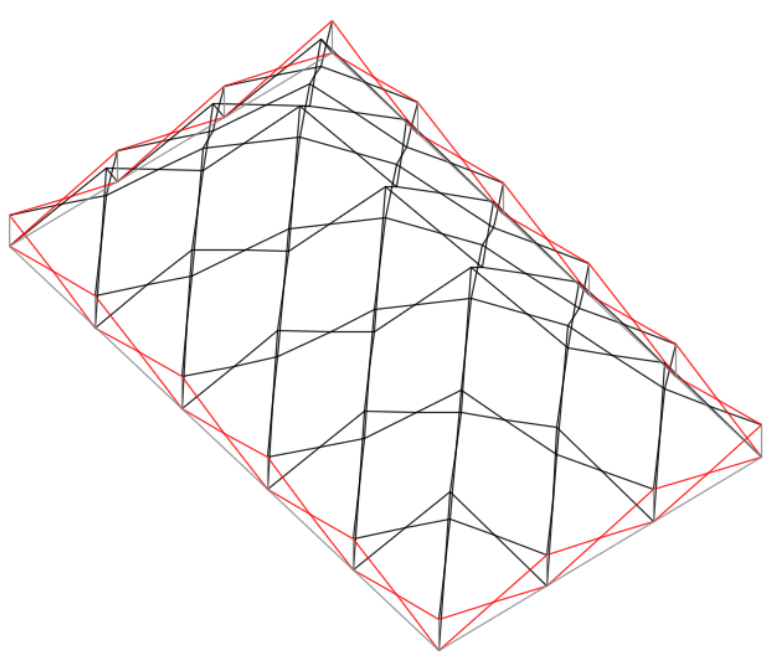

Figure 18: Mesh with Corrected Initial Slope

In the same way as in the previous examples, where the blades were tied horizontally, it is also possible to propose tying them in the direction of the maximum slope, although always with composite scissors, giving rise to a combination using a type of transverse portico. These elements arise from the need to complete the free edges in on-the-bias cylindrical deployable structures with two gables (Figure 19) or with vault-shaped edges (Figure 20), as otherwise the end would be indented.

Obviously, the layout of the edge blades can be adjusted in the way indicated in Figure 15.

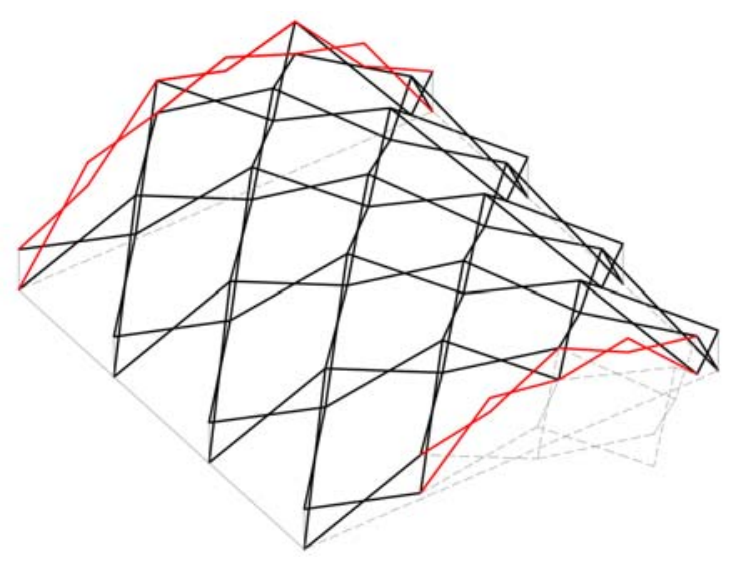

Figure 19: Ends of the Free Edges of a Cylindrical on-the-Bias Deployable Structure with and without Overhang.

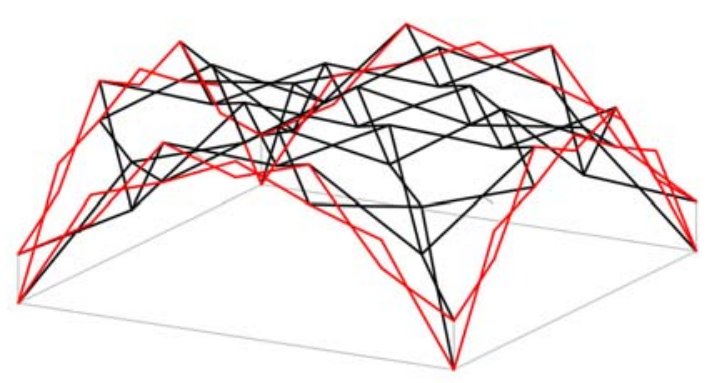

Figure 20: Ends of the free edges of a deployable structure in the shape of a cross vault.

\section{POLAR AND TRANSLATIONAL UNIT COMBINATIONS.}

One limitation of the previous proposals is that the ground clearance is totally conditioned by the slope and length of the starting module bars. A solution to this problem can be found by combining the previous deployment structures with polar modules around the edge.

Polar units are those in which the contact nodes between the scissors are located above the radii of the directrix circumference, or -generalizing for any curve-, those in which the contact nodes between blades are located at each point above the radii of curvature of the guide. Translational units are those in which the contact nodes between scissors are located on straight lines that are parallel to each other, usually vertically. If these definitions are accepted, both groups have one element in common: the meshes of horizontal flat blades, inasmuch as these meshes comply with both definitions (taking into account that parallel lines intersect at infinity). Other authors [12] prefer to consider flat meshes as a separate type, classifying the modules as straight (flat meshes), curved (polar) and oblique (translational).

With the exception of the use of flat meshes, polar and translational combinations have rarely been used. These include the annular deployable proposal formulated by You \& Pellegrino in 1993, consisting of two rings made up of translation modules interconnected with polar modules [3] -p. $20-$.

Recently, applying the criteria outlined in [7], our team has solved the combination of polar and translational modules in the same guideline within two-way deployable meshes (Figure 21). The 
solution was implicit in [7], but is now shown explicitly. To construct the mesh, polar modules, translational modules and concurrence modules are used, while transversally they are all straight modules.

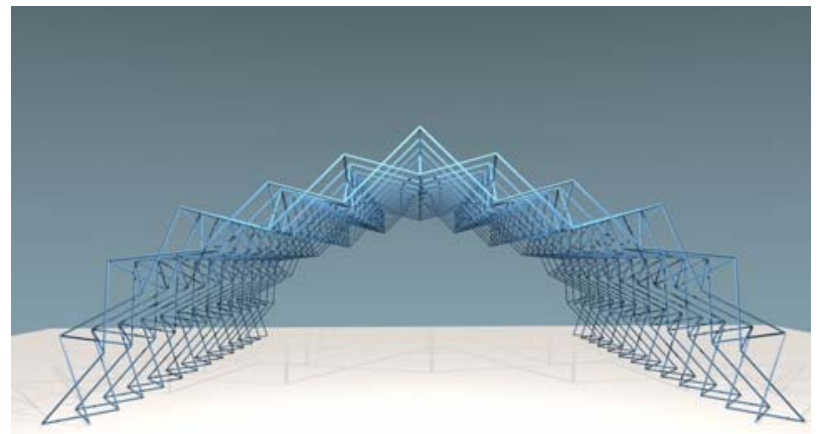

Figure 21: Cylindrical Deployable Structure Made Using Polar and Translational Modules

Here the aim is to gain height by raising the on-thebias deployable structure with a series of polar modules starting from the rectangular perimeter. A first option is that the polar modules maintain the $45^{\circ}$ planes of the on-the-bias deployable structure, which gives rise to elegant solutions with quite simple layouts, but in which the polar units of the supports only share one node (Figure 22). Obviously, the height and inclination of the perimeter polar modules are conditioned by complying with the folding conditions.

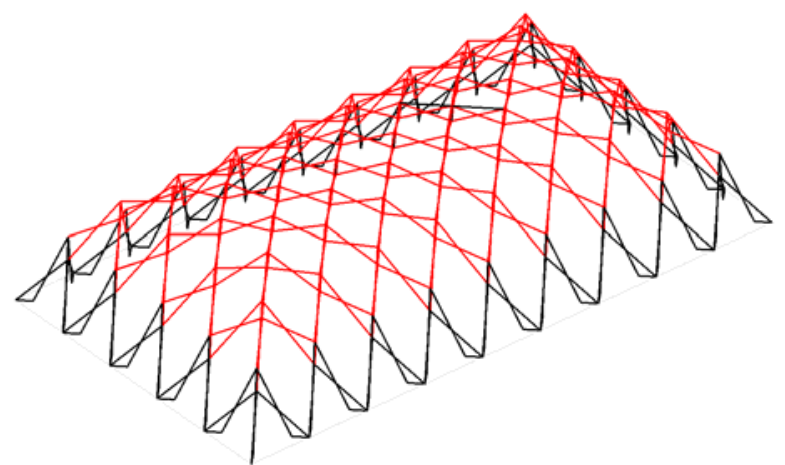

Figure 22. Rectangular Pavilion Vault on-the-Bias Deployable Structure on Perimeter Polar Units

Another possibility is to arrange the polar modules in parallel to the sides of the ground plan, as part of an approach that is a consequence of the implementation of the perimeter scissors: the aim is to complete the polar modules outlined with the perimeter bracing.

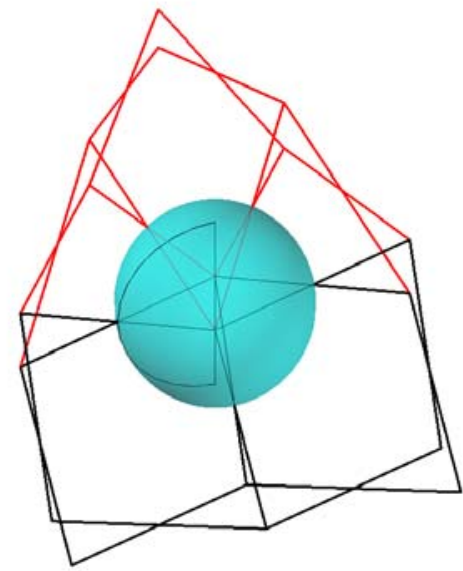

Figure 23: Revolution Ellipsoid that Expresses the Second Condition of Foldability in 3-D.

Based on the solutions in which the slope of the translational starting modules has been adjusted so that the perimeter is formed by single scissors, the ellipsoid of revolution is defined that contains the possible positions of the scissor joints of the blades that meet the conditions of foldability (Figure 23), and therefore of the straight section (ellipse), meaning it is enough to decide the number of polar modules to be placed as a transition to the ground (one or two in the images) in order to have the directions that will establish the position of the scissor-type joints. In the images it has been applied to 4-gable solutions with one and two polar modules, and to rectangular pavilion vaults with an initial polar module (Figures 24 and 25).

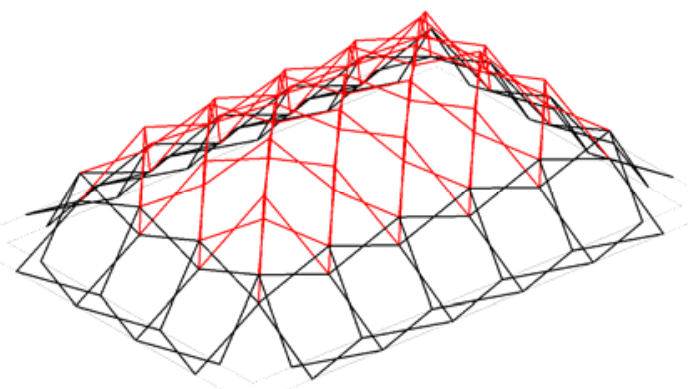

Figure 24: On-the-Bias Deployable Structure Adjusted on One Row of Perimeter Polar Modules 


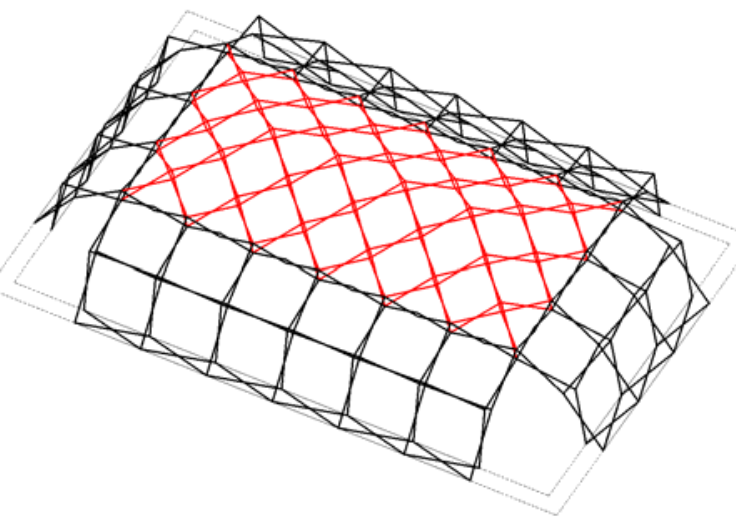

Figure 25: Adjusted on-the-Bias Deployable Structure on Two Rows of Polar Perimeter Modules

A second alternative is that the arrangement with the transverse dimension is carried out by the added polar modules, starting with the on-the-bias deployable structure with the perimeter enclosed by composite scissors, and the adjustment is carried out in the layout of the polar modules including an asymmetric polar module, which is extended as necessary to carry out the adjustment required by the transverse blade, as shown in Figure 26.

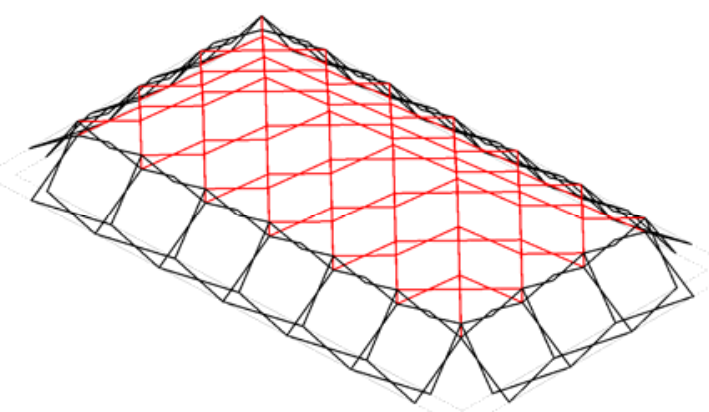

Figure 26: On-the-Bias Deployable Structure on a Row of Polar Adjustment Modules

In the current state of the proposals the corners remain open. With respect to the nodes, a simple Y node resolves the connection between the perimeter blades and bias mesh bars; the inclusion of a horizontal direction in the bias mesh requires more complex nodes with unfolded articulations for the perimeter.

\section{MODIFICATIONS TO THE EXTERNAL APPEARANCE}

Although the rectangular ground plan allows for compact layouts, the exterior appearance of these foldouts is too neutral, lacking expressiveness.
Indicating an entrance, including a light, or allowing for other types of groupings are architectural demands that can be indispensable in certain types of uses, such as dining rooms or spaces for religious worship, for which the possibilities -at least theoretical- of the on-the-bias deployable structures have been explored.

It is simple to create a symmetry in one of the modules that forms a support, thereby obtaining an overhang that marks an entry point while maintaining perfect foldability, or to extend the symmetry to all the lateral starting modules to achieve a continuous overhang, which with a global symmetry (and therefore maintaining its foldability conditions) becomes two enclosures with a covered central corridor, as can be seen in the image.

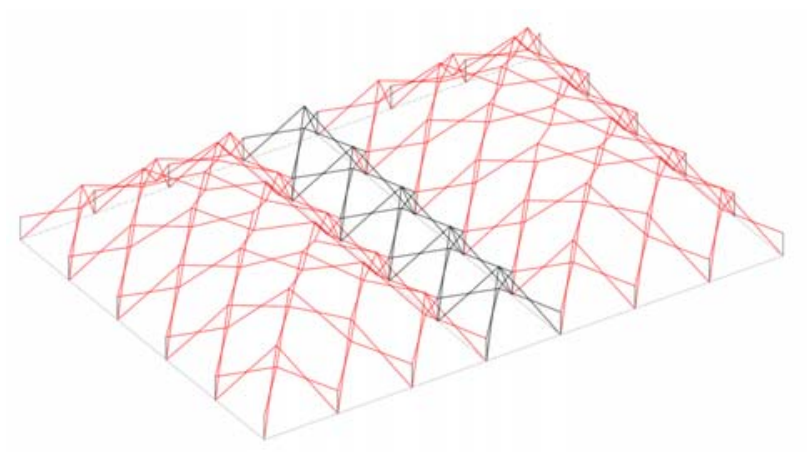

Figure 27: Combinations of on-the-Bias Deployable Structures

It is also possible to replicate the process to create the end caps of the headers on the long sides and thus obtain a roof configuration with new angles that make it possible to obtain points of the edge that do not provide support, in order to create higher entrances, or to resolve L- or T-shaped ground plans with the same deployable structure (Figure 28). Although the formation of enclosures by symmetry is a possibility of translational modules in general, the adaptation to $\mathrm{L}$ or $\mathrm{T}$ ground plans is exclusive to on-the-bias deployable structures. In this way, the problem of the deployable structures noted by Escrig -[6] p.75- regarding the lack of modularity in the ground plan is solved.

Going into more detail, the folding conditions are maintained if, from a set of four previously selected contiguous nodes, blades are erected with positive and negative slopes that duplicate the surrounding surface and allow for the creation of roof openings for skylights for example - with which to enliven the resulting space. In this way, envelopes are 
obtained that are separated from the anodyne profile of the marquee, and introduce new design possibilities.

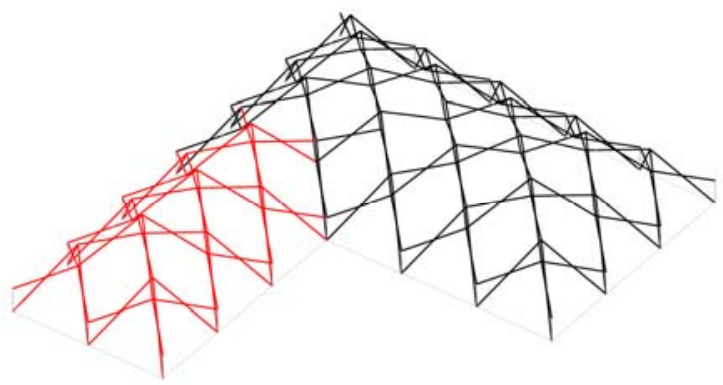

Figure 28: On-the-Bias Deployable Structure with an L-Shaped Ground Plan

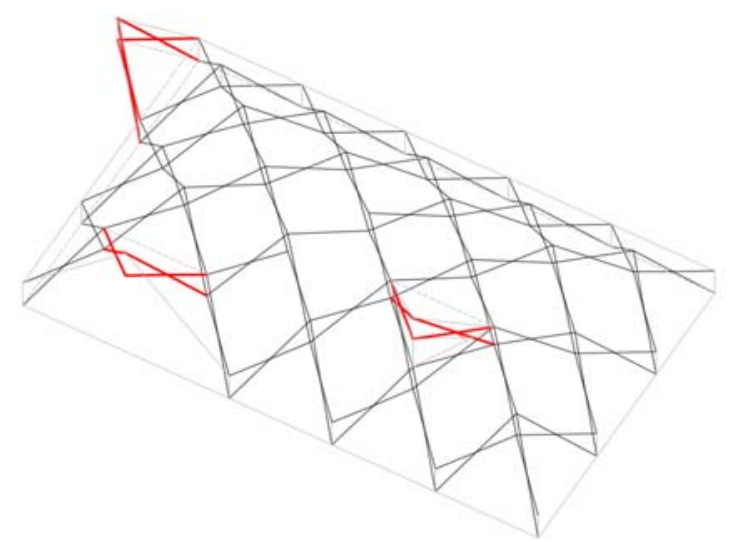

Figure 29: Modified on-the-Bias Deployable Structure

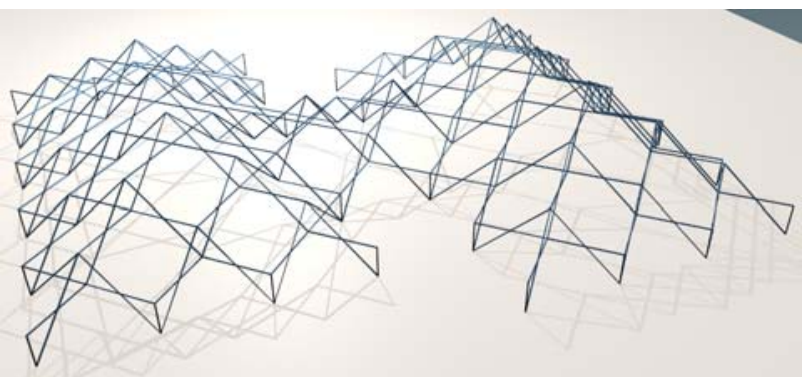

Figure 30: Modularity of on-the-Bias Deployable Structures

These possibilities were explored in a model, verifying the perfect functioning of the proposed designs: perimeter cantilevers, deployable structures with a T-shaped floor plan, or high entrances, using standard bars and nodes. Only the proposal of a double folding surface - in order to be able to make a vertical window on deck for example - posed certain problems, given that it requires the use of solutions with different nodes so that they do not collide with each other during the unfolding process, and to give the bars that define the external surface an extra length so that the interiors pass through inside during the folding and unfolding process. In addition, it is necessary to define a specific criterion for the assembly of the bars to allow for deployment, which -in the applied solution- requires the insertion of a bar and to do without the redundant intermediate articulation of the affected blade, in order to avoid complex crossings that pose a challenge to its flexibility.

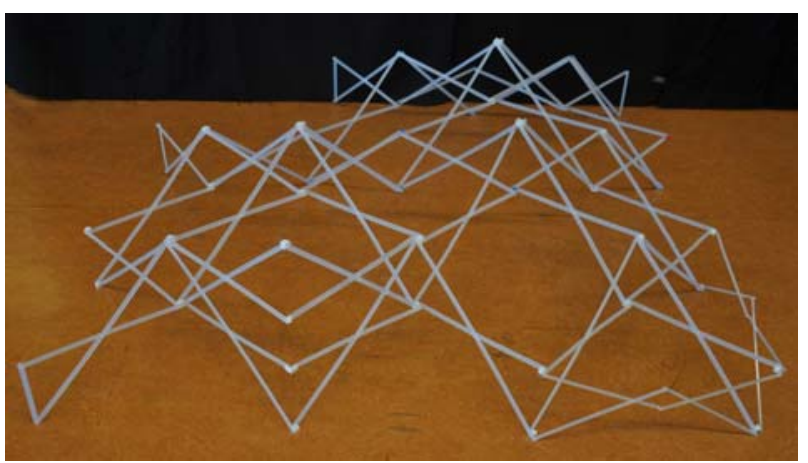

Figure 31: Model of on-the-Bias Deployable Structure with L-Shaped Ground Plan

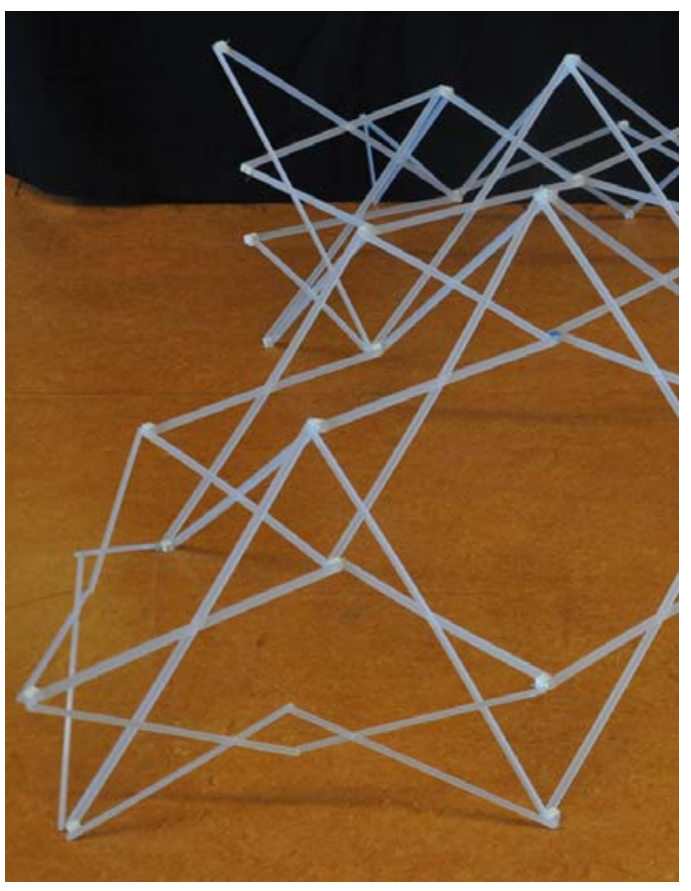

Figure 32: Model with a Vertically Duplicated Module 


\section{CONCLUSIONS}

- Extremities are a problem for any deployable structure with a cylindrical guideline on a rectangular floor plan that seeks to close a space for which some solutions have been tested.

- On-the-bias deployable structures solve this problem using known geometries, such as the foursided roof or those with the shape of a rectangular pavilion vault, since they allow the structure to be supported along the entire perimeter of the floor plan.

- They also have the advantage of eliminating the dichotomy between supporting and bracing blades, an aspect that gives them greater strength and rigidity than cylindrical blades, as has been proven with the models and calculations that were made.

- These deployable structures have a degree of geometric flexibility that allows the manipulation of the roof profile within certain limits.

- The models that were built revealed that the corner blades are unloaded, which makes it difficult to maintain the shape of the structure. This problem can be solved by incorporating bracing scissors in the corner arranged at the edges (at $45^{\circ}$ with the structure blades) that can be extended to all of the perimeters.

- The combination of polar and translation meshes has been resolved, both within the same geometry cylindrical deployable structures- and in the perimeter of the on-the-bias deployable structures, generating a Y support, with perpendicular directions to the edges.

- The system solves the modularity of the deployable structures in terms of their floor plan, providing solutions for $\mathrm{L}$ - and $\mathrm{T}$ - plans, and allowing a multitude of combinations. It also allows for the introduction of architectural nuances, such as overhangs, patios and skylights.

\section{ACKNOWLEDGMENTS}

This research was carried out as a part of the Spanish Research Project on Deployable and Modular Constructions for Situations of Humanitarian Catastrophe, CODEMOSCH (Reference BIA2016-79459-R), funded by the Spanish Ministry of Industry, Energy, and Competitiveness (MINECO).

\section{REFERENCES}

[1] F. Escrig, J. P. Valcárcel and J. Sánchez, "Deployable Structures Squared in Plan: Design and Construction" in Spatial Structures: Heritage, Present and Future. Proceeding of the IASS International Symposium 1995, Milano, Italy, June 5-9 1995. G.C. Giuliani Ed. SG Editoriali 1995 pp. 483-492.

[2] L. Sánchez, "Geometric models for expandable structures" in Mobile and Rapidly Assembling Structures II. Proceeding of the $2^{\text {nd }}$ MARAS 1996, Seville, Spain, June 17-20, 1996, Escrig \& Brebbia, Ed. Computational Mechanics Publications, Southampton, 1996, pp. 93-102.

[3] N. De Temmerman, "Design and Analysis of Deployable Bar Structures for Mobile Architectural Applications". Ph.D. dissertation, Department of Architectural Engineering Sciences. Vrije Universiteit Brussel, Brussels, 2007.

[4] C. Hernández and W. Zalewski, Pabellón Itinerante de Exposiciones TaraTara, Estado de Falcón, Venezuela, 2005 (based on the ESTRAN 1 Protoype developed by Hernández Merchán and Zalewski, 19872000. IDEC - FAU - UCV.

[5] C.h. Hernández, "New Ideas on Deployable Structures" in Mobile and Rapidly Assembling Structures II. Proceeding of the $2^{\text {nd }}$ MARAS 1996, Seville, Spain, June 17-20, 1996, Escrig \& Brebbia, Ed. Computational Mechanics Publications, Southampton, 1996, pp. 63-72.

[6] F. Escrig, Modular, Ligero, Transformable: un Paseo por la Arquitectura Ligera Móvil. Sevilla, Secretariado de publicaciones de la Universidad de Sevilla, 2012.

[7] M.J. Freire-Tellado et al., "Scissor-Hinged Deployable Structures for Inclined Roofs", Informes de la Construcción, vol. 70-555, pp. \#-\#, September-October 2019 (DOI currently unavailable)

[8] R. Ramos, "New Geometry Deployable Structure of X-frame", in New proposals for transformable architecture, engineering and design: in honour of Emilio Pérez Piñero: 
Proceedings of the first conference transformable 2013, Seville, Spain, F. Escrig and J. Sánchez, Eds. Starbooks, 2013, pp. 8991

[9] J. Pérez-Valcárcel et al. "Expandable bar structures for emergency situations" in $4^{\text {th }}$ International Conference on Structures and Architecture: Proceeding of the 4th ICSA 2019, Lisbon, Portugal, July 24-26, P. Cruz Ed. Publisher, Year. pp. \#-\#. (DOI currently unavailable)

[10] J. Begiristain, “XXX, Sistemas Estructurales Desplegables para Infraestructuras de Intervención Urbana Autoconstruidas", Ph.D. dissertation, Departamento de Arquitectura, Universidad del País Vasco, San Sebastián, 2015.

[11] M. Muñoz-Vidal, "ARTIC 3 Computer program” Spain. DL: C-79-12. RPI: C-4662011. ISBN: 978-84-92794-52-2. DOI 10.13140-RG.2.1.3288.1041

[12] J. P. Valcárcel et alii "Cálculo no lineal de estructuras desplegables de barras curvas" in 21 Congreso de Métodos Numéricos en Ingeniería. Proceeding of the $2^{\text {nd }}$ SEMNI 1993, A Coruña, Spain, June 7-11, 1993, F. Navarrina, M. Casteleiro Eds. Artes Gráficas Torres SA, 1993, Vol 1. pp 400-409.

The data in red are not available, as the corresponding references are currently in press. 